University of Wollongong

Research Online

Faculty of Engineering and Information

Faculty of Engineering and Information

Sciences - Papers: Part B

Sciences

2018

\title{
A Disturbance Observer-Based Robust Controller Design for Systems with Right Half Plane Zeros and Poles
}

\author{
Emre Sariyildiz \\ University of Wollongong, emre@uow.edu.au \\ Ilhan Mutlu \\ IAV Automotive Engineering \\ Rahim Mutlu \\ University of Wollongong, rmutlu@uow.edu.au
}

Follow this and additional works at: https://ro.uow.edu.au/eispapers1

Part of the Engineering Commons, and the Science and Technology Studies Commons

Research Online is the open access institutional repository for the University of Wollongong. For further information contact the UOW Library: research-pubs@uow.edu.au 


\title{
A Disturbance Observer-Based Robust Controller Design for Systems with Right Half Plane Zeros and Poles
}

\begin{abstract}
This paper analytically derives the bandwidth limitations of Disturbance Observer (DOB) when plants have Right Half Plane (RHP) zero(s) and pole(s). If the plant is non-minimum phase, then the bandwidth of DOB should be set at a lower value than its upper bound to improve the robust stability and performance. If the plant is unstable, then the bandwidth of DOB should be set at a higher value than its lower bound to achieve the robust stability. The upper and lower bounds are analytically derived by using Poisson integral formula. It is shown that the bandwidth limitation of DOB is directly related to the locations of the RHP zero(s) and pole(s) and becomes more severe as they get close each other. A minimum phase approximation of the non-minimum phase nominal plant model is proposed by using Genetic Algorithm (GA) to tackle the internal stability problem of the DOB-based robust control systems. Simulation results are given to verify the proposed robust controllers.
\end{abstract}

\section{Keywords}

controller, robust, right, observer-based, half, disturbance, plane, zeros, poles, systems, design

\section{Disciplines}

Engineering | Science and Technology Studies

\section{Publication Details}

Sariyildiz, E., Mutlu, I. \& Mutlu, R. (2018). A Disturbance Observer-Based Robust Controller Design for Systems with Right Half Plane Zeros and Poles. European Journal of Control, 41 53-62. 


\title{
A Disturbance Observer-Based Robust Controller Design for Systems with Right Half Plane Zeros and Poles
}

\author{
Emre Sariyildiz ${ }^{1 *}$, İlhan Mutlu ${ }^{2}$, and Rahim Mutlu ${ }^{1}$ \\ ${ }^{1}$ School of Mechanical, Materials, Mechatronic and Biomedical Engineering, \\ University of Wollongong, NSW, 2522, Australia \\ emre@uow.edu.au; rmutlu@uow.edu.au \\ ${ }^{2}$ Development Center Chemnitz/Stollberg, IAV GmbH, 09366 Stollberg, Germany \\ Ilhan.Hyusein.Hasan@iav.de \\ "Corresponding Author: Emre Sariyildiz; email: emre@ uow.edu.au; Phone: +61242213319
}

Abstract- This paper analytically derives the bandwidth limitations of Disturbance Observer (DOB) when plants have Right Half Plane (RHP) zero(s) and pole(s). If the plant is non-minimum phase, then the bandwidth of DOB should be set at a lower value than its upper bound to improve the robust stability and performance. If the plant is unstable, then the bandwidth of DOB should be set at a higher value than its lower bound to achieve the robust stability. The upper and lower bounds are analytically derived by using Poisson integral formula. It is shown that the bandwidth limitation of DOB is directly related to the locations of the RHP zero(s) and pole(s) and becomes more severe as they get close each other. A minimum phase approximation of the non-minimum phase nominal plant model is proposed by using Genetic Algorithm (GA) to tackle the internal stability problem of the DOB-based robust control systems. Simulation results are given to verify the proposed robust controllers.

Index Terms: Disturbance Observer; Non-minimum Phase Systems; Robust Control; Robustness and Performance Trade-off; Unstable Systems.

\section{INTRODUCTION}

It is a well-known fact that plants with RHP zero(s) and pole(s), i.e., non-minimum phase and unstable plants, have several constraints, such as bandwidth limitation and achievable sensitivity reduction, in the design of feedback control systems [1, 2]. As it is pointed out by G. Stein in 1989 Bode lecture, control of such systems is quantifiably harder than minimum phase stable systems, and special consideration is required due to their fundamental characteristics such as local stability [3, 4]. The control problem of non-minimum phase and/or unstable plants becomes more severe when they suffer from plant uncertainties. Goodwin et al. showed that the average performance of such systems in the presence of model uncertainties can significantly depart from the best achievable nominal performance, i.e., the performance without model uncertainties [5]. Therefore, the robust control problem of plants with RHP zero(s) and pole(s) is a very challenging problem in the literature. 
DOB is a robust control tool that is used to estimate plant uncertainties and external disturbances [6, 7]. In DOB-based robust control, the estimations of disturbances are fed back through control input in an inner-loop so that the robustness of the system is simply obtained by cancelling disturbances with their estimations. An outer-loop controller is designed to achieve performance goals by considering only the nominal plant dynamics as DOB cancels disturbances in the inner-loop [8, 9]. A DOB-based robust controller has two-degrees-of-freedom; i.e., its robustness and performance can be independently adjusted in the inner and outer loops, respectively. Although DOB has been applied to many different applications, such as industrial automation, robotics and automotive, in the last three decades, it still suffers from insufficient control techniques [10] - [12]. Therefore, in general, its applications highly depend on designers' own experiences [13, 14].

Since a DOB can precisely estimate disturbances within the frequency range of its low-pass-filter (LPF), the robustness against disturbances of a DOB-based control system can be simply improved by increasing the bandwidth of the LPF [7, 14]. However, it is limited by practical design constraints, such as noise and sampling time, in real implementations. The bandwidth limitations of DOB become more severe when plants have RHP zero(s) and/or pole(s) [15, 16]. Furthermore, the inverse of the nominal plant model, which is required in the design of DOB, causes internal stability problem in DOB-based robust control if the plant has non-minimum phase zero(s) [17] - [19]. Therefore, DOB-based robust control of systems with RHP zero(s) and/or pole(s) is also quantifiably harder than that of minimum phase stable systems.

This paper proposes DOB-based robust controllers for plants with RHP zero(s) and/or pole(s). The design limitations of the robust controller, i.e., the upper and lower bounds of the bandwidth of DOB, are analytically derived by using Poisson integral formula. It is shown that if the plant is non-minimum phase and the bandwidth of DOB is set at a higher value than its upper bound, then the peaks of the sensitivity and co-sensitivity functions may dramatically increase; i.e., the robust stability and performance may dramatically deteriorate. On the other hand, if the plant is unstable, then the peaks of the sensitivity and cosensitivity functions can be simply lessened by increasing the bandwidth of the LPF of DOB. The upper and lower bounds of the bandwidth of DOB are directly related to the locations of the RHP zero(s) and pole(s), and the bandwidth limitations become stricter as they get close each other. When DOB is applied to a non-minimum phase system, it suffers from internal stability problem as the inverse of the nominal plant model is required in the design of DOB. In this paper, the internal stability problem is tackled by proposing a minimum phase approximate model for the non-minimum phase nominal plant. The approximate nominal plant model is obtained by minimizing the differences between the magnitude and phase responses of minimum and non-minimum phase plant models within the limited frequency range of DOB. When DOB is applied to an unstable system, the outer-loop controller is designed to stabilize the inner-loop. Therefore, a feedforward controller is proposed to adjust the performance of the robust controller in this paper. The validity of proposals is verified by giving 
simulation results of the robust control of a non-minimum phase plant with unstructured uncertainties and an unstable plant with parametric uncertainties.

The rest of the paper is organized as follows. In section II, feedback control limitations of systems with RHP zero(s) and pole(s) are briefly explained. In section III, a DOB-based robust control system is presented, and the bandwidth limitations of DOB are analytically derived when plants have RHP zero(s) and pole(s). In section IV, the minimum phase approximate model of a non-minimum phase plant is obtained by using Genetic Algorithm (GA). In section V, simulation results of the robust control of non-minimum phase and unstable systems are presented. The paper ends with conclusion given section VI.

\section{Limitations OF CONTROL SYSTEMS With Right HALF PlANE ZERO(S) AND POLE(S)}

In this section, the fundamental limitations of control systems with RHP zero(s) and pole(s) are briefly explained. The reader is invited to refer to [2,] [20-22] for further details.

\section{A. Performance and Phase-Margin Limitations of Control Systems with RHP Zero(s) and Pole(s)}

It is a well-known fact that RHP zero(s) and pole(s) cause undershoot and overshoot in feedback control systems, respectively. They are directly related to the bandwidth of the closed loop system, and its constraints are defined by using

$$
\begin{aligned}
& w_{B} \leq \frac{2.1991 z_{R H P}}{\log \left(1-0.9\left(y_{\text {undershoot }}\right)^{-1}\right)} \\
& w_{B} \geq \frac{2.1991 p_{R H P}}{\log \left(10\left(y_{\text {overshoot }}-0.9\right)\right)}
\end{aligned}
$$

where $w_{B}$ represents the bandwidth of the closed-loop system, $z_{R H P}$ and $p_{R H P}$ represent the RHP zero and pole of the system, respectively, and $y_{\text {undershoot }}$ and $y_{\text {overshoot }}$ represent the infimum and supremum of the system's step response, respectively [2, 20].

To obtain a good phase-margin, the crossover frequency of a non-minimum phase system should be smaller than its RHP zero, i.e.,

$$
w_{C} \leq \gamma z_{R H P}
$$

where $w_{C}$ represents the crossover frequency, and $0.5 \leq \gamma \leq 1[20]$.

\section{B. Bode and Poisson Integral Formulas}

The fundamental feedback control limitation of a stable minimum phase system can be described by using Bode integral formula as follows: 


$$
\int_{0}^{\infty} \log (|S(j w)|) d w=0
$$

where $S(j w)$ represents the sensitivity function of the system in frequency domain, $j$ represents complex number and $w$ represents frequency [21].

Eq. (4) shows that the sensitivity function of a control system cannot be freely shaped. For example, as the robustness against disturbances is improved by decreasing the sensitivity function at low frequencies, the system may become more noise sensitive since the peak value of the sensitivity function $(\sup (\log (|S(j w)|))>0 \mathrm{~dB})$ may increase to satisfy Eq. (4). However, the noise sensitivity may not be a serious problem as low peak values of the sensitivity function can still balance the integral equation in infinite frequency range.

Let us now consider systems with RHP zero(s) and pole(s) and define an open loop transfer function by using

$$
L(s)=\tilde{L}(s) B_{S}^{-1}(s) B_{T}(s)
$$

where $\tilde{L}(s)$ represents a minimum phase stable transfer function, and $B_{S}(s)=\prod_{i=1}^{k} \frac{p_{i}-s}{\bar{p}_{i}+s}$ and $B_{T}(s)=\prod_{i=1}^{l} \frac{z_{i}-s}{\bar{z}_{i}+s}$ represent Blaschke products in which $z_{i}$ and $p_{i}$ are the RHP zero and pole of $L(s)$, respectively, and $\bar{z}_{i}$ and $\bar{p}_{i}$ are conjugates of $z_{i}$ and $p_{i}$, respectively [20].

The fundamental feedback control limitations of systems with RHP zero and/or pole are more severe than that of minimum phase stable systems. They can be similarly described by using Poisson integral formula as follows [2, 20]:

$$
\begin{aligned}
& \int_{-\infty}^{\infty} \log (|S(j w)|) W_{z}(j w) d w=\pi \log \left(\left|B_{S}^{-1}\left(z_{R H P}\right)\right|\right) \\
& \int_{-\infty}^{\infty} \log (|T(j w)|) W_{p}(j w) d w=\pi \log \left(\left|B_{T}^{-1}\left(p_{R H P}\right)\right|\right)
\end{aligned}
$$

where $S(j w)=\frac{1}{1+L(j w)}$ and $T(j w)=1-S(j w)$ respectively represent the sensitivity and co-sensitivity functions of the system in frequency domain; $z_{R H P}=\sigma_{z}+j w_{z}$ represents the RHP zero of the system in which $\sigma_{z}$ and $w_{z}$ are real and imaginary parts of $z_{R H P}$, respectively; $p_{R H P}=\sigma_{p}+j w_{p}$ represents the RHP pole of the system in which $\sigma_{p}$ and $w_{p}$ are real and imaginary parts of $p_{R H P}$, respectively; $B_{S}^{-1}(s)$ and $B_{T}^{-1}(s)$ are the inverse of the Blaschke products which are given in Eq. (5); and $W_{z}(j w)=\frac{\sigma_{z}}{\sigma_{z}^{2}+\left(w_{z}-w\right)^{2}}$ and $W_{p}(j w)=\frac{\sigma_{p}}{\sigma_{p}^{2}+\left(w_{p}-w\right)^{2}}$ are weighting functions. 


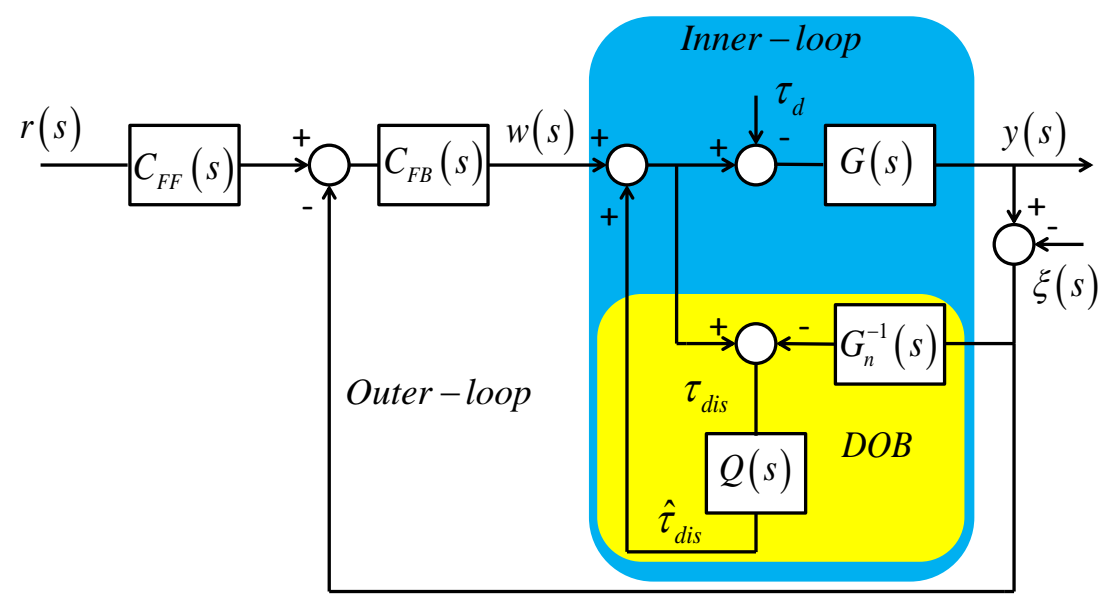

Fig. 1. Block diagram of a DOB-based robust control system.

Against the Bode integral formula, the Poisson integral equations should balance in a finite frequency range as the weighting functions $W_{z}(j w)$ and $W_{p}(j w)$ decay with the increasing frequency. Therefore, the peak values of the sensitivity and cosensitivity functions can be high, e.g., the system may become very sensitive to noise, as the robustness against disturbances is improved by decreasing the sensitivity function at low frequencies.

\section{DESIGN OF Disturbance ObSERVER-BASED Robust CONTROL SySTEMS}

In this section, DOB-based robust controllers are proposed for systems with RHP zero(s) and pole(s) by considering their fundamental feedback control limitations. Let us first explain the general structure of a DOB-based robust control system.

\section{A. A DOB-based Robust Control System}

Block diagram of a DOB-based robust control system is illustrated in Fig. $1[7,14]$. In this figure, $G(s)$ and $G_{n}(s)$ represent uncertain and nominal plant models, respectively; $r(s)$ and $\xi(s)$ represent reference and noise exogenous inputs, respectively; $y(s)$ represents output of the system; $\tau_{d}$ represents exogenous disturbance input; $\tau_{d i s}$ represents disturbances due to model mismatch and $\tau_{d}$, i.e., $\tau_{d i s}=\left(G^{-1}(s)-G_{n}^{-1}(s)\right) y(s)+\tau_{d}$ when $\xi(s)=0 ; \hat{\tau}_{\text {dis }}$ represents the estimation of $\tau_{d i s} ; Q(s)$ represents the LPF of DOB; and $C_{F B}(s)$ and $C_{F F}(s)$ represent the feedback and feed-forward outer-loop controllers, respectively.

Let us first assume that $C_{F F}(s)=1$. The open-loop, sensitivity and co-sensitivity transfer functions of a DOB-based robust control system can be directly derived from Fig. 1 as follows:

Inner Loop:

$$
L_{i}(s)=\frac{G(s) Q(s)}{G_{n}(s)(1-Q(s))}
$$




$$
S_{i}=\frac{1}{1+L_{i}(s)}=\frac{G_{n}(s)(1-Q(s))}{G_{n}(s)(1-Q(s))+G(s) Q(s)}, \quad T_{i}=\frac{L_{i}(s)}{1+L_{i}(s)}=\frac{G(s) Q(s)}{G_{n}(s)(1-Q(s))+G(s) Q(s)}
$$

Outer Loop:

$$
\begin{gathered}
L_{o}(s)=\frac{C_{F B}(s) G(s) G_{n}(s)}{G_{n}(s)(1-Q(s))+G(s) Q(s)} \\
S_{o}=\frac{1}{1+L_{o}(s)}=\frac{G_{n}(s)(1-Q(s))+G(s) Q(s)}{G_{n}(s)(1-Q(s))+G(s) Q(s)+G(s) G_{n}(s) C_{F B}(s)}, \quad T_{o}=\frac{L_{o}(s)}{1+L_{o}(s)}=\frac{G(s) G_{n}(s) C_{F B}(s)}{G_{n}(s)(1-Q(s))+G(s) Q(s)+G(s) G_{n}(s) C_{F B}(s)}
\end{gathered}
$$

where $L_{\bullet}, S_{\bullet}$ and $T_{\bullet}$ represent the open loop, sensitivity and co-sensitivity transfer functions, respectively.

If the bandwidth of the LPF of DOB goes to infinite and/or the frequency of disturbances goes to zero, i.e., $Q(s) \rightarrow 1$, then

Inner-loop

$$
L_{i}(s) \rightarrow \infty, S_{i} \rightarrow 0 \text { and } T_{i} \rightarrow 1
$$

Outer Loop:

$$
L_{o}(s) \rightarrow C_{F B}(s) G_{n}(s), S_{o} \rightarrow \frac{1}{1+C_{F B}(s) G_{n}(s)} \text { and } T_{o} \rightarrow \frac{C_{F B}(s) G_{n}(s)}{1+C_{F B}(s) G_{n}(s)}
$$

Eq. (12) shows that a DOB-based robust control system can precisely suppress constant disturbances in the inner-loop. However, many control systems are influenced by not only constant but also variable disturbances. Although the robustness against variable disturbances can be simply improved by increasing the bandwidth of the LPF of DOB, it is limited by practical design constraints such as sampling time and noise. In other words, a DOB-based control system can provide high-robustness within a limited frequency range which is determined by the bandwidth of the LPF of DOB [14].

Eq. (13) shows that $C_{F B}(s)$ can be designed by considering only the nominal model of the plant so that the outer-loop transfer functions can be shaped to achieve performance requirements. A DOB-based robust control system has a 2-DOF control structure; i.e., its robustness and performance can be independently tuned via DOB and performance controllers in the inner and outer loops, respectively [7].

\section{B. Limitations of Non-Minimum Phase Systems in DOB-based Robust Control}

For the sake of simplicity, let us assume that $Q(s)=\frac{g}{s+g}$ is the first order LPF of DOB. Let us also assume that $G(s)$ and $G_{n}(s)$ are the uncertain and nominal plant models which have RHP zero(s) and $\hat{G}_{n}(s)$ is the approximate nominal plant 
model of $G(s)$ which has stable inverse [19]. $G(s)$ can be defined in terms of $G_{n}(s)\left(\right.$ or $\left.\hat{G}_{n}(s)\right)$ and a multiplicative unstructured uncertainty as follows:

$$
G(s)=G_{n}(s)\left(1+\Delta W_{T}(s)\right)=\hat{G}_{n}(s) r_{\text {err }}(s)\left(1+\Delta W_{T}(s)\right)
$$

where $r_{e r r}(s)=G_{n}(s)\left(\hat{G}_{n}(s)\right)^{-1} ;\|\Delta(s)\| \leq 1$; and $W_{T}(s)$ is the weighting transfer function of the multiplicative unstructured uncertainty. The approximate minimum phase nominal plant model of $G(s)$ is used to satisfy internal stability of the robust control system [19].

If Eq. (14) and the first order LPF of DOB are substituted into Eq. (8-11), then the inner and outer loop transfer functions are derived as follows:

Inner Loop:

$$
\begin{gathered}
L_{i}(s)=\frac{g r_{\text {err }}(s)\left(1+\Delta W_{T}(s)\right)}{s} \\
S_{i}(s)=\frac{s}{s+g r_{\text {err }}(s)\left(1+\Delta W_{T}(s)\right)}, \quad T_{i}(s)=\frac{g r_{\text {err }}(s)\left(1+\Delta W_{T}(s)\right)}{s+g r_{\text {err }}(s)\left(1+\Delta W_{T}(s)\right)}
\end{gathered}
$$

Outer Loop:

$$
\begin{gathered}
L_{o}(s)=\frac{C_{F B}(s) G(s)(s+g)}{s+g r_{e r r}(s)\left(1+\Delta W_{T}(s)\right)} \\
S_{o}(s)=\frac{s+g r_{e r r}(s)\left(1+\Delta W_{T}(s)\right)}{s+g r_{e r r}(s)\left(1+\Delta W_{T}(s)\right)+C_{F B}(s) G(s)(s+g)}, \quad T_{o}(s)=\frac{C_{F B}(s) G(s)(s+g)}{s+g r_{e r r}(s)\left(1+\Delta W_{T}(s)\right)+C_{F B}(s) G(s)(s+g)}
\end{gathered}
$$

When a DOB-based robust controller is applied to a non-minimum phase system, the bandwidth limitations of the LPF of DOB can be analytically derived by using the following theorems.

Theorem 1: If $G(s)$ has a RHP zero at $z_{R H P}$, then the DOB-based robust control system should satisfy the following design constraint to obtain a good phase margin.

$$
\left.\left|g r_{e r r}(j w)\left(1+\Delta W_{T}(j w)\right)\right|\right|_{w=w_{c}} \leq \gamma z_{R H P}
$$

where $w_{C}$ represents the crossover frequency, and $0.5 \leq \gamma \leq 1$ (see Eq. (3)).

Proof: Eq. (19) is derived by directly applying Eq. (15) to Eq. (3). 
Theorem 2: If $G(s)$ has a RHP zero at $z_{R H P}$, then the bandwidth of DOB should hold Eq. (20) to satisfy the robustness and performance goals which are respectively defined by $\left|S_{i}(j w)\right| \leq \alpha_{S_{i}}, \forall w \leq w_{S_{i}}$ and $\left|T_{i}(j w)\right| \leq \alpha_{T_{i}}, \forall w \geq w_{T_{i}}$.

$$
\begin{aligned}
& \frac{\left(1-\alpha_{S_{i}}\right) k^{-1} z_{R H P} \psi_{2}}{\alpha_{S_{i}}\left|r_{e r r}(j w)\left(1+\Delta W_{T}(j w)\right)\right|} \leq g \leq \frac{\alpha_{T_{i}} k z_{R H P} \psi_{1}}{\left.\left(1-\alpha_{T_{i}}\right)\left|r_{e r r}(j w)\left(1+\Delta W_{T}(j w)\right)\right|\right|_{w=w_{T_{i}}}} \\
& \psi_{1}=\tan \left(\frac{\log \left(1+\alpha_{T_{i}}\right)\left(\pi-2 \vartheta\left(w_{T_{i}}\right)\right)+2 \log \left(\max _{w_{S_{i}} \leq w \leq w_{T_{i}}}\left(\left|S_{i}(j w)\right|\right)\right) \vartheta\left(w_{T_{i}}\right)}{2\left(\log \left(\max _{w_{S_{i}} \leq w \leq w_{T_{i}}}\left(\left|S_{i}(j w)\right|\right)\right)+\log \left(\alpha_{S_{i}}{ }^{-1}\right)\right)}-\frac{\pi \log \left(\left|B_{S}^{-1}\left(z_{R H P}\right)\right|\right)}{2\left(\log \left(\max _{w_{S_{i}} \leq w \leq w_{T_{i}}}\left(\left|S_{i}(j w)\right|\right)\right)+\log \left(\alpha_{S_{i}}{ }^{-1}\right)\right)}\right), \\
& \psi_{2}=\tan \left(\frac{\log \left(\left(1+\alpha_{T_{i}}\right)^{-1}\right) \pi+2\left(\log \left(\alpha_{S_{i}}^{-1}\right)+\log \left(\max _{w_{S_{i}} \leq w \leq w_{T_{i}}}\left(\mid S_{i}(j w)\right)\right)\right) \vartheta\left(w_{S_{i}}\right)}{2\left(\log \left(\max _{w_{S_{i}} \leq w \leq w_{T_{i}}}\left(\left|S_{i}(j w)\right|\right)\right)+\log \left(\left(1+\alpha_{T_{i}}\right)^{-1}\right)\right)}+\frac{\pi \log \left(\left|B_{S}^{-1}\left(z_{R H P}\right)\right|\right)}{\left.2\left(\log \left(\max _{w_{S} \leq w \leq w_{W_{i}}}\left(\left|S_{i}(j w)\right|\right)\right)+\log \left(\alpha_{S_{i}}^{-1}\right)\right)\right)},\right. \\
& k=w_{T_{i}} w_{S_{i}}^{-1} \text { and } \vartheta\left(w_{\bullet}\right)=\int_{0}^{w_{0}} W_{z}(w) d w=\int_{0}^{w_{0}} \frac{z_{R H P}}{z_{R H P}^{2}+w^{2}} d w=\arctan \left(\frac{w_{\bullet}}{z_{R H P}}\right) .
\end{aligned}
$$

where

Proof: If $\left|T_{i}(j w)\right| \leq \alpha_{T_{i}}, \forall w \geq w_{T_{i}}$, then $\left|S_{i}(j w)\right| \leq 1+\alpha_{T_{i}}, \forall w \geq w_{T_{i}}$ [20]. Let us consider the Poisson integral formula which is given in Eq. (6). If $\left|S_{i}(j w)\right| \leq \alpha_{S_{i}}, \forall w \leq w_{S_{i}}$ and $\left|T_{i}(j w)\right| \leq \alpha_{T_{i}}, \forall w \geq w_{T_{i}}$ are applied to Eq. (6), then

$$
\log \left(1+\alpha_{T_{i}}\right)\left(\int_{-\infty}^{-w_{T_{i}}} W_{z}(w) d w+\int_{w_{T_{i}}}^{\infty} W_{z}(w) d w\right)+\log \left(\alpha_{S_{i}}\right) \int_{-w_{S_{i}}}^{w_{S_{S}}} W_{z}(w) d w+\log \left(\max _{w_{S_{i}} \leq w \leq w_{i_{i}}}\left(\left|S_{i}(j w)\right|\right)\right)\left(\int_{-w_{i_{i}}}^{-w_{S_{i}}} W_{z}(w) d w+\int_{w_{S_{i}}}^{w_{T_{i}}} W_{z}(w) d w\right) \geq \pi \log \left(\left|B_{S}^{-1}\left(z_{R H P}\right)\right|\right)
$$

If the integral of the weighting function $\left(W_{z}(w)\right)$ is applied to Eq. (21), then

$$
\begin{gathered}
\log \left(\max _{w_{S_{i}} \leq w \leq w_{i_{i}}}\left(\left|S_{i}(j w)\right|\right)\right) \geq \log \left(\alpha_{S_{i}}^{-1}\right) \frac{2 \vartheta\left(w_{S_{i}}\right)}{2\left(\vartheta\left(w_{T_{i}}\right)-\vartheta\left(w_{S_{i}}\right)\right)}+\log \left(\left(1+\alpha_{T_{i}}\right)^{-1}\right) \frac{\pi-2 \vartheta\left(w_{T_{i}}\right)}{2\left(\vartheta\left(w_{T_{i}}\right)-\vartheta\left(w_{S_{i}}\right)\right)}+\left|B_{S}^{-1}\left(z_{R H P}\right)\right| \frac{\pi}{2\left(\vartheta\left(w_{T_{i}}\right)-\vartheta\left(w_{S_{i}}\right)\right)} \\
\vartheta\left(w_{S_{i}}\right) \leq \frac{\log \left(1+\alpha_{T_{i}}\right)\left(\pi-2 \vartheta\left(w_{T_{i}}\right)\right)+2 \log \left(\max _{w_{S_{i}} \leq w \leq w_{T_{i}}}\left(\left|S_{i}(j w)\right|\right)\right) \vartheta\left(w_{T_{i}}\right)}{2\left(\log \left(\max _{w_{S_{i}} \leq w \leq w_{T_{i}}}\left(\left|S_{i}(j w)\right|\right)\right)+\log \left(\alpha_{S_{i}}{ }^{-1}\right)\right)}-\frac{\pi \log \left(\left|B_{S}^{-1}\left(z_{R H P}\right)\right|\right)}{2\left(\log \left(\max _{w_{S_{i}} \leq w \leq w_{T_{i}}}\left(\left|S_{i}(j w)\right|\right)\right)+\log \left(\alpha_{S_{i}}{ }^{-1}\right)\right)} \\
\vartheta\left(w_{T_{i}}\right) \geq \frac{\log \left(\left(1+\alpha_{T_{i}}\right)^{-1}\right) \pi+2\left(\log \left(\alpha_{S_{i}}^{-1}\right)+\log \left(\max _{w_{S_{i}} \leq w \leq w_{T_{i}}}\left(\left|S_{i}(j w)\right|\right)\right)\right) \vartheta\left(w_{S_{i}}\right)}{2\left(\log \left(\max _{w_{S_{i}} \leq w \leq w_{T_{i}}}\left(\left|S_{i}(j w)\right|\right)\right)+\log \left(\left(1+\alpha_{T_{i}}\right)^{-1}\right)\right)}+\frac{\pi \log \left(\left|B_{S}^{-1}\left(z_{R H P}\right)\right|\right)}{2\left(\log \left(\max _{w_{S_{i}} \leq w \leq w_{T_{i}}}\left(\left|S_{i}(j w)\right|\right)\right)+\log \left(\left(1+\alpha_{T_{i}}\right)^{-1}\right)\right)}
\end{gathered}
$$


$\psi_{1}$ and $\psi_{2}$ can be derived by using Eq. (23) and Eq. (24). The constraints on the LPF of DOB can be derived by using $\left|S_{i}(j w)\right| \leq \alpha_{S_{i}}, \forall w \leq w_{S_{i}}$ and $\left|T_{i}(j w)\right| \leq \alpha_{T_{i}}, \forall w \geq w_{T_{i}}$ as follows:

$$
g \geq \frac{1-\alpha_{S_{i}}}{\alpha_{S_{i}}} \frac{w}{\left|r_{\text {err }}(s)\left(1+\Delta W_{T}(s)\right)\right|} \forall w \leq w_{S_{i}} \leq z_{R H P} \psi_{1} \text { and } g \leq \frac{\alpha_{T_{i}}}{1-\alpha_{T_{i}}} \frac{w}{\left|r_{\text {err }}(s)\left(1+\Delta W_{T}(s)\right)\right|} \forall w \geq w_{T_{i}} \geq z_{R H P} \psi_{2}
$$

Let us assume that $w_{T_{i}}=k w_{S_{i}}$ where $k>1$. Hence, Eq. (25) can be rewritten as follows:

$$
\frac{1-\alpha_{S_{i}}}{\alpha_{S_{i}}} \frac{k^{-1} w_{T_{i}}}{\left|r_{e r r}\left(k^{-1} w_{T_{i}}\right)\left(1+\Delta W_{T}\left(k^{-1} w_{T_{i}}\right)\right)\right|} \leq g \leq \frac{\alpha_{T_{i}}}{1-\alpha_{T_{i}}} \frac{k w_{S_{i}}}{\left|r_{e r r}\left(k w_{S_{i}}\right)\left(1+\Delta W_{T}\left(k w_{S_{i}}\right)\right)\right|}
$$

If $w_{S_{i}} \leq z_{R H P} \psi_{1}$ and $w_{T_{i}} \geq z_{R H P} \psi_{2}$ are substituted into Eq. (26), then Eq. (20) is derived.

In Theorem 1 and Theorem 2, the upper and lower bounds of the bandwidth of DOB are analytically derived to achieve the robustness and performance goals which are defined by $\left|S_{i}(j w)\right| \leq \alpha_{S_{i}}, \forall w \leq w_{S_{i}}$ and $\left|T_{i}(j w)\right| \leq \alpha_{T_{i}}, \forall w \geq w_{T_{i}}$. For example, as $\alpha_{S_{i}}$ is decreased and/or $w_{S_{i}}$ is increased, the robustness of the system improves. However, the sensitivity and co-sensitivity functions of a DOB-based control system cannot be freely shaped due to the RHP zero of the plant.

Eq. (22) shows that the peak of the sensitivity function is in the frequency range of $w_{S_{i}} \leq w \leq w_{T_{i}}$. The peak value is directly related to the shapes of the sensitivity and co-sensitivity functions, i.e., $\alpha_{S_{i}}, w_{S_{i}}, \alpha_{T_{i}}$ and $w_{T_{i}}$. For example, as the robustness of the control system is improved by decreasing the sensitivity function at low frequencies, i.e., using lower $\alpha_{S_{i}}$, or keeping the sensitivity function low in a larger frequency range, i.e., using higher $w_{S_{i}}$, the peak of the sensitivity function increases. Therefore, there are lower and upper bounds for $\alpha_{S_{i}}$ and $w_{S_{i}}$, respectively. Eq. (22) also shows that the peak value of the sensitivity function is larger if the plant has RHP pole as well. Higher peak values appear as the RHP pole gets closer to the RHP zero.

Eq. (25) shows that the sensitivity and co-sensitivity functions can be directly shaped by tuning the bandwidth of the LPF of DOB. For example, as it is increased, lower values of the sensitivity function can be achieved in a larger frequency range, i.e., the robustness against disturbances of the control system is simply improved. However, as shown in Eq. (22), the bandwidth of DOB is limited by the RHP zero of the plant. 
Theorem 2 is conservative due to the ideal sectionally constant constraints of the sensitivity function. Therefore, the peak value of the sensitivity function can be lower than the derived one in Theorem 2 . The conservatism can be lessened by using more realistic constraints for the sensitivity and co-sensitivity transfer functions [22].

The performance controller can be designed by considering the performance constraint given in Eq. (1).

\section{Limitations of Unstable Systems in DOB-based Robust Control}

Let us now consider systems with RHP poles. If the nominal plant model is unstable, then the inner-loop of the DOB-based robust control system is also unstable when disturbances are precisely suppressed. Therefore, the outer-loop feedback controller should be designed to stabilize the overall system. In this paper, a feed-forward controller, which is illustrated as $C_{F F}(s)$ in Fig. 1, is proposed to achieve the performance goals of systems with RHP poles.

When a DOB-based robust controller is applied to an unstable system, the bandwidth limitations of the LPF of DOB can be analytically derived by using the following theorem.

Theorem 3: If $G(s)$ has a RHP pole at $p_{R H P}$, then the bandwidth of DOB should hold Eq. (20) and Eq. (21) to satisfy the performance goal which is defined by $\left|T_{o}(j w)\right| \leq \alpha_{T_{o}}, \forall w \geq w_{T_{o}}$.

$$
\begin{gathered}
\frac{|C G(j w)(j w+g)|}{\left|w+g\left(1+\Delta W_{T}(j w)\right)\right|} \leq \frac{\alpha_{T_{o}}}{\left|1-\alpha_{T_{o}}\right|}, \forall w \geq p_{R H P} \psi \\
\frac{\left|C G\left(j p_{\text {RHP }} \psi\right)\left(j p_{R H P} \psi+g\right)\right|}{\left|j p_{\text {RHP }} \psi+g\left(1+\Delta W_{T}\left(j p_{\text {RHP }} \psi\right)\right)+C G\left(j p_{\text {RHP }} \psi\right)\left(j p_{\text {RHP }} \psi+g\right)\right|} \geq \alpha_{T_{o}}
\end{gathered}
$$

where $\psi=\tan \left(\frac{\pi\left(\log \left(\alpha_{T_{o}}^{-1}\right)+\log \left(\left|B_{T}^{-1}\left(p_{R H P}\right)\right|\right)\right)}{2\left(\log \left(\alpha_{T_{o}}^{-1}\right)+\log \left(\left\|T_{i}\right\|_{\infty}\right)\right)}\right)$.

Proof: If $\left|T_{o}(j w)\right| \leq \alpha_{T_{o}}, \forall w \geq w_{T_{o}}$ is applied to Eq. (7), then

$$
\log \left(\alpha_{T_{o}}\right)\left(\int_{-\infty}^{-w_{T_{o}}} W_{p}(w) d w+\int_{w_{T_{o}}}^{\infty} W_{p}(w) d w\right)+\log \left(\|T\|_{\infty}\right) \int_{-w_{T_{o}}}^{w_{T_{p}}} W_{p}(w) d w \geq \pi \log \left(\left|B_{T}^{-1}\left(p_{R H P}\right)\right|\right)
$$

If the integral of the weighting function is applied to Eq. (29), then

$$
\log \left(\left\|T_{o}\right\|_{\infty}\right) \geq \log \left(\alpha_{T_{o}}^{-1}\right) \frac{\pi-2 \vartheta\left(w_{T_{o}}\right)}{2 \vartheta\left(w_{T_{o}}\right)}+\log \left(\left|B_{T}^{-1}\left(p_{R H P}\right)\right|\right) \frac{\pi}{2 \vartheta\left(w_{T_{o}}\right)}
$$




$$
\vartheta\left(w_{T_{o}}\right) \geq \frac{\pi\left(\log \left(\alpha_{T_{o}}^{-1}\right)+\log \left(\left|B_{T}^{-1}\left(p_{R H P}\right)\right|\right)\right)}{2\left(\log \left(\alpha_{T_{o}}^{-1}\right)+\log \left(\left\|T_{o}\right\|_{\infty}\right)\right)}
$$

where $\vartheta\left(w_{T_{o}}\right)=\int_{0}^{w_{T_{o}}} W_{p} d w=\int_{0}^{w_{T_{o}}} \frac{p_{R H P}}{p_{R H P}^{2}+w^{2}} d w=\arctan \left(\frac{w_{T_{o}}}{z_{R H P}}\right)$.

$\psi$ is derived by using Eq. (31). It shows that $w_{T_{o}}>p_{R H P} \psi$. The constraint on the LPF of DOB and the outer-loop stabilizing controller can be derived by using $\left|T_{o}(j w)\right| \leq \alpha_{T_{o}}, \forall w \geq w_{T_{o}}$ as follows:

$$
\frac{|C G(j w)(j w+g)|}{\left|j w+g\left(1+\Delta W_{T}(j w)\right)+C G(j w)(j w+g)\right|} \leq \alpha_{T_{o}}, \forall w \geq w_{T_{o}}
$$

Eq. (27) and Eq. (28) are derived by applying $w_{T_{o}} \geq p_{R H P} \psi$ to Eq. (32).

Eq. (30) shows that the peak of the co-sensitivity function increases as $\alpha_{T_{o}}$ and $w_{T_{o}}$ decrease. To improve the robust stability and performance of the system, the co-sensitivity function should be smoothly decreased by limiting either $\alpha_{T_{o}}$ or $w_{T_{o}}$. Eq. (30) also shows that the peak value of the co-sensitivity function is larger if the plant has RHP zero as well. Higher peak values appear as the RHP zero gets closer to the RHP pole.

Eq. (32) shows that the co-sensitivity function can be directly shaped by tuning the bandwidth of DOB and/or outer-loop stabilizing controller. Eq. (27) and Eq. (28) show that the bandwidth of DOB and the outer-loop stabilizing controller should have upper bounds to achieve the performance goal which is defined by $\left|T_{o}(j w)\right| \leq \alpha_{T_{o}}, \forall w \geq w_{T_{o}}$. However, if their upper bounds are not high enough, i.e., if $\alpha_{T_{o}}$ gets smaller in a narrow frequency range, then high peak values of the co-sensitivity function may appear as shown in Eq. (30). Either the bandwidth of DOB or the gain of the outer-loop stabilizing controller should be increased to improve the robust stability and performance of the system. Increasing the gain of the outer-loop stabilizing controller causes higher energy consumption than increasing the bandwidth of DOB. Therefore, shaping the cosensitivity function via DOB improves energy efficiency.

Theorem 3 is also conservative due to the ideal sectionally constant constraints of the co-sensitivity function. The conservatism can be similarly lessened by using more realistic constraints for the co-sensitivity transfer function [22].

The performance controller can be designed by considering the performance constraint given in Eq. (2).

\section{Minimum Phase Approximate Model of a Non-Minimum Phase Plant}


To achieve internal stability, the minimum phase approximate model of the non-minimum phase nominal plant is used in the design of DOB. Similar solutions can be found in the literature; e.g., the approximate model is obtained by using least mean square error in [19]. In general, the approximation problem of non-minimum phase systems is hard to solve since an infinite dimensional stable filter is required in principle to get an exact inverse system model [23]. In this paper, the approximate minimum phase model is obtained by using GA so that the effect of the model mismatch between $G_{n}(s)$ and $\hat{G}_{n}(s)$, i.e. $r_{\text {err }}(s)$, is minimized within a limited frequency range. This frequency range is determined by the bandwidth limitation of the robust controller, i.e., DOB. The approximate minimum phase plant model is designed by using GA as follows:

Let us consider a non-minimum phase nominal plant model, which has RHP zeros, by using

$$
G_{n}(s)=\frac{N(s)}{D(s)}
$$

where $N(s)$ and $D(s)$ represent the numerator and denominator polynomials of $G_{n}(s)$, respectively.

Let us assume that $n$ and $d(n \leq d)$ are respectively the degrees of $N(s)$ and $D(s)$ polynomials and $l \leq n$ is the number of RHP zeros which cause the non-minimum phase behavior. Let us also define an error polynomial to derive the approximate minimum phase model of the non-minimum phase plant by using

$$
e(s)=N(s) A(s)-B(s)
$$

where $A(s)$ and $B(s)$ are the polynomials with left-half-plane (LHP) zeros, and the degrees of $A(s)$ and $B(s)$ are $m$ and $m+n$, respectively.

$$
\text { If } e(s) \cong 0, \text { then }
$$

$$
N(s) \cong \frac{B(s)}{A(s)}
$$

where $B(s) / A(s)$ is a non-casual minimum phase transfer function.

Let us define a quadratic integral performance index by using Eq. (34).

$$
\begin{gathered}
\min : \quad \int_{0}^{\min \left(\operatorname{Re}\left(z_{\text {RHP }}\right)\right)}\left\{\lambda_{\text {amp }}|e(j w)|^{2}+\lambda_{\text {phase }}(\arg (e(j w)))^{2}\right\} d w, \\
\text { s.t. : }\left\{\begin{array}{c}
A(s), B(s) \text { and } s+g r_{\text {err }}(s) \text { are Hurwitz polynomials } \\
-\pi \leq \arg (e(j w)) \leq \pi
\end{array}\right\}
\end{gathered}
$$

where $\lambda_{\text {amp }}$ and $\lambda_{\text {phase }}$ represent the relative weighting factors for the magnitude and phase errors, respectively. Since the bandwidth of the robust control is limited by the location of RHP zero(s) as shown in Theorem 2, the optimization range is 
bounded by $0 \leq w<\min \left(\operatorname{Re}\left(z_{R H P}\right)\right)$ in Eq. (36). In other words, the model mismatched between $G_{n}(s)$ and $\hat{G}_{n}(s)$ is minimized within the frequency range of DOB's bandwidth.

Eq. (36) is not convex so the analytical solution of the optimization problem is not straightforward. In this study, GA which is a powerful evolutionary algorithm in terms of global numerical optimization is preferred to determine the coefficients of the approximation polynomials $[24,25]$.

\section{CASE Studies: DOB-BASEd Robust Control of Systems With RHP ZERo AND Pole}

In this section, DOB-based robust controllers are designed for systems with RHP zero and pole. The validity of the proposals is verified by giving simulation results.

\section{A. DOB-based Robust Control of a System with a RHP Zero}

Let us consider a non-minimum phase system by using

$$
G(s)=G_{n}(s)\left(1+\Delta W_{T}(s)\right)
$$

where $G_{n}(s)=\frac{-s+25}{s^{2}+15 s+50}, W_{T}(s)=\frac{3.75 s+400}{s+1500}$ and $|\Delta| \leq 1$

Let us first design the approximate minimum phase nominal plant model of $G(s)$ to achieve internal stability. GA calculations are carried out by using the Global Optimization Toolbox of Matlab $® 2014 \mathrm{~b}$. A Matlab routine is created to implement the cost function and the constraints that is given Eq. (36). In the problem formulation, GA parameters are selected as follows: population size is 20 , maximum number of generations is 100 , fitness scaling is rank, selection function is stochastic uniform, crossover type is scattered, mutation function is Gaussian and stopping criteria is $10^{-6}$.

If it is assumed that $\lambda_{\text {amp }}=1, \lambda_{\text {phase }}=1, A(s)=a_{1} s+a_{0}$ and $B(s)=s^{2}+b_{1} s+b_{0}$, then the minimum phase nominal plant model is obtained by using Eq. (36) and GA as follows:

$$
\hat{G}_{n}(s)=\frac{s^{2}+350 s+25}{14.04 s+1} \frac{1}{s^{2}+15 s+50}
$$

where $\frac{B(s)}{A(s)}=-\frac{s^{2}+350 s+25}{14.04 s+1} \cong s-25$.

Fig. 2 illustrates the frequency responses of the RHP zero polynomial, i.e., $s-25$, and its non-casual minimum phase approximation, i.e., $B(s) / A(s)=-\left(s^{2}+350 s+25\right) / 14.04 s+1$. It is clear from the figure that similar frequency responses are 


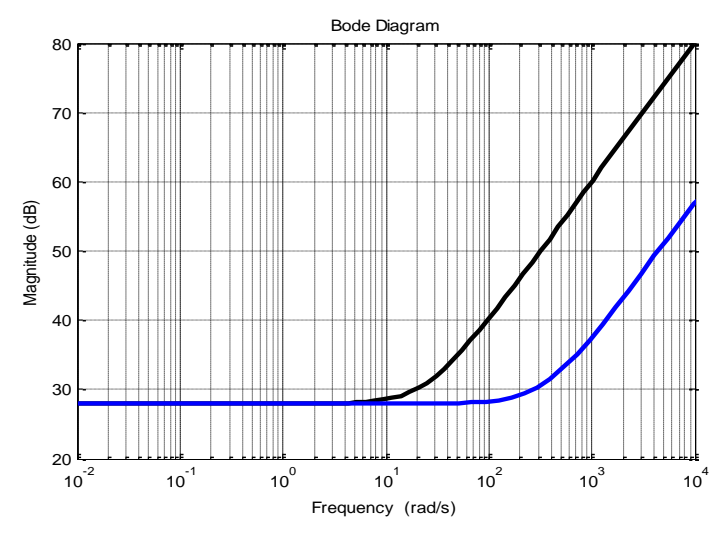

a) Amplitude Response

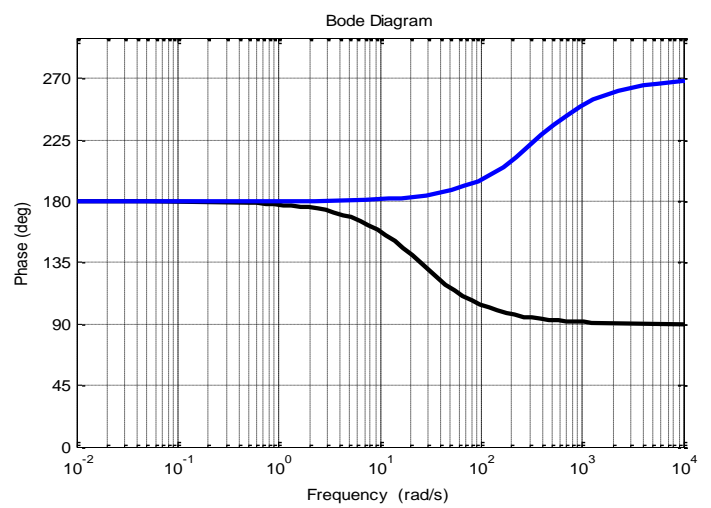

b) Phase Response

Fig. 2. Bode diagrams of the RHP zero polynomial (Black Curve) and its nan-casual minimum phase approximation (Blue Curve).

achieved within the limited frequency range, i.e., $25 \mathrm{rad} / \mathrm{s}$. Therefore, the effect of the model mismatch between $G_{n}(s)$ and $\hat{G}_{n}(s)$ can be ignored within the bandwidth of DOB.

Let us now consider the bandwidth limitations of DOB by using Theorem 1 and Theorem 2 . The former directly shows that the bandwidth of DOB should be smaller than $25 \mathrm{rad} / \mathrm{s}$ to achieve a good phase margin. The sensitivity and co-sensitivity function constraints should be determined to derive the bandwidth limitations of DOB via Theorem 2. Let us assume that the robustness and performance goals of the control system are $\left|S_{i}(j w)\right| \leq 1 / \sqrt{2}, \forall w \leq w_{S_{i}}$ and $\left|T_{i}(j w)\right| \leq 1 / 2, \forall w \geq w_{T_{i}}$, respectively, and the peak of the sensitivity function is $\left\|S_{i}\right\|_{\infty}=2$. The bandwidth limitation of DOB is derived by using Eq. (20) as follows:

$$
6.46 \leq g \leq 21.27 \text { and } w_{S_{i}} \leq 34 \mathrm{rad} / \mathrm{s}
$$

The frequency range of the control goal, i.e., $w_{S_{i}}$ and $w_{T_{i}}$, depends on practical applications. For example, if the control system is noise sensitive or has unmodeled dynamics at low frequencies, then lower $w_{T_{i}}$ should be used.

Fig. 3 illustrates the frequency responses of the sensitivity and co-sensitivity functions of the inner-loop. As the bandwidth of DOB is increased, the robustness against disturbances is improved by decreasing the sensitivity function in a larger frequency range. However, the robust stability and noise sensitivity deteriorate as the peak values of the sensitivity and cosensitivity functions are increased. Small changes in the bandwidth of DOB may cause high peaks in the frequency responses of $S_{i}(s)$ and $T_{i}(s)$. Fig. 3 shows that the control goal and robust stability can be achieved if the bandwidth of DOB is between 8 and $11 \mathrm{rad} / \mathrm{s}$. The difference between Fig. 3 and Eq. (39) is due to the conservatism in Theorem 2. More realistic bandwidth limitations can be derived by lessening the conservatism. 
Bode Diagram

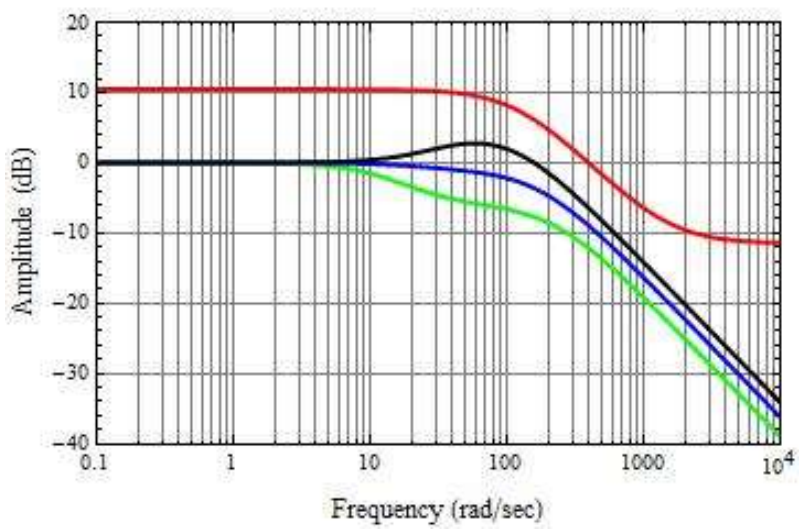

a) Frequency responses of the co-sensitivity function.
Bode Diagram

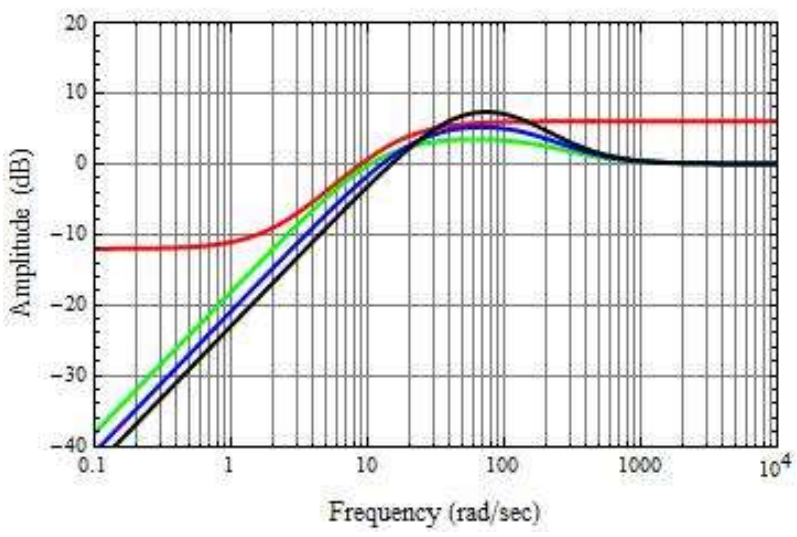

b) Frequency responses of the sensitivity function.

Fig. 3. Frequency responses of the inner-loop sensitivity and co-sensitivity functions. Red curves: Inverses of weighting functions; green curves: $g=8 \mathrm{rad} / \mathrm{s}$; blue curves: $\mathrm{g}=11 \mathrm{rad} / \mathrm{s}$; black curves: $\mathrm{g}=14 \mathrm{rad} / \mathrm{s}$

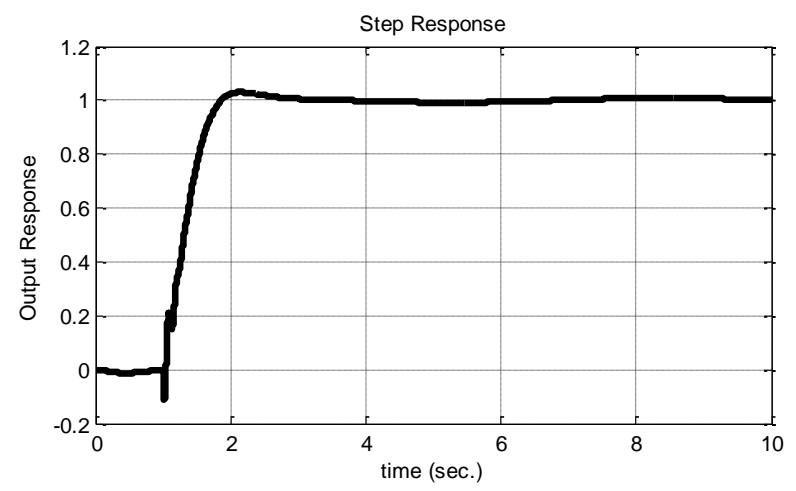

Fig. 4. Step response of the robust control system when the plant has a RHP zero at 25.

Fig. 4 illustrates the step response of the robust control system when it is disturbed by $\tau_{d}=\sin (t)+u(t-2)$. The outer loop controller $C_{F B}(s)=1.125+0.1 s+6 / s$ is designed by considering the bandwidth constraint of the system which is given in Eq. (1). It is clear from the figure that the system can precisely follow the step reference input by cancelling the disturbances via DOB. The undershoot due to RHP zero is suppressed by satisfying Eq. (1). It can be lessened by decreasing the bandwidth of the closed-loop system, yet the performance of the step response deteriorates. There is a trade-off between the undershoot response and performance of the system as shown in Eq. (1).

\section{B. DOB-based Robust Control of a System with a RHP Pole}

Let us consider the uncertain and nominal plant models by using

$$
G(s)=\frac{\delta_{n 0}}{\delta_{d 2} s^{2}-\delta_{d 1} s} \text { and } G_{n}(s)=\frac{1}{s^{2}-s}
$$

where $0.1 \leq \delta_{n 0} \leq 1.4,0.6 \leq \delta_{d 1} \leq 2$ and $0.1 \leq \delta_{d 2} \leq 1.6$, are the uncertain parameters, which have upper and lower bounds, of the system. 


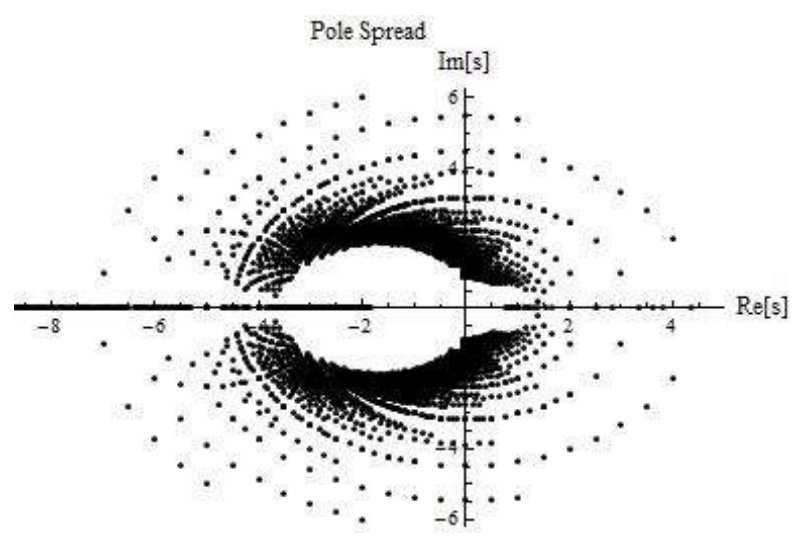

a) $C_{F B}(s)=10+6 s$

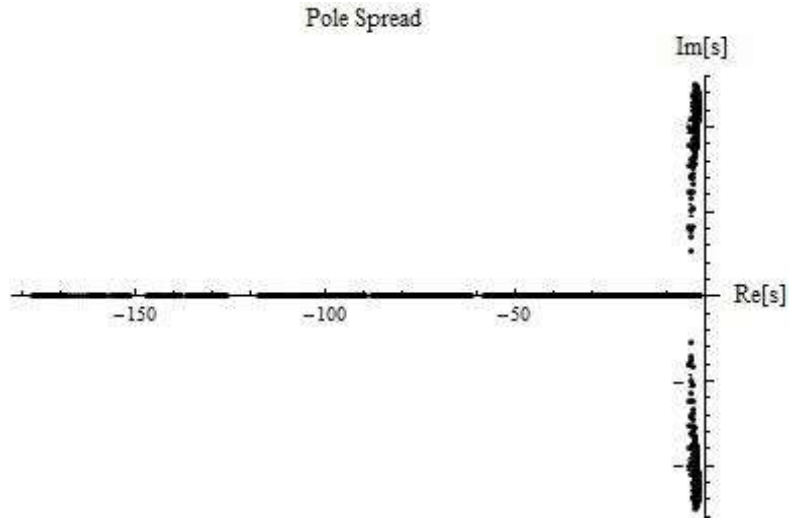

b) $C_{F B}(s)=20+40 s$

Fig. 5. Pole spread of the closed-loop system when different stabilizing controllers are implemented.

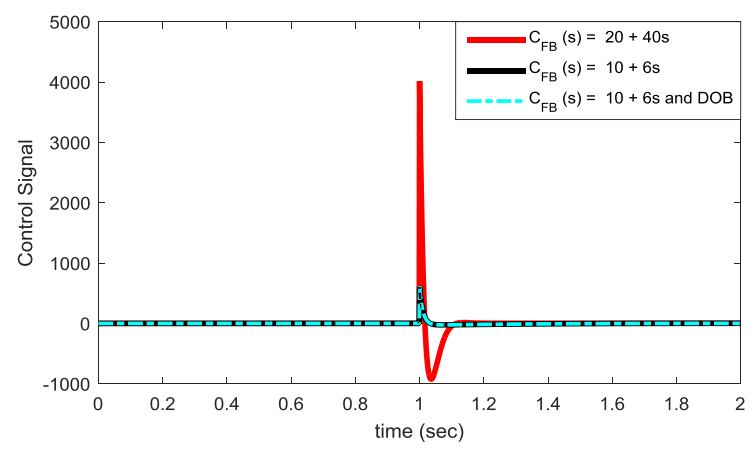

Fig. 6. Control signals for different controller designs.

Let us first ignore DOB-based robust control and stabilize the unstable plant by using only the outer-loop feedback controller. Two stabilizing feedback controllers can be designed for the nominal plant model of the system by using

$$
\begin{aligned}
& C_{F B}(s)=10+6 s \\
& C_{F B}(s)=20+40 s
\end{aligned}
$$

Fig. 5 illustrates the pole spreads of the closed-loop system when Eq. (41) and Eq. (42) are used to stabilize the unstable plant with parametric uncertainties. It is clear from the figure that the robust stability cannot be achieved when low control gains are used. Although the robust stability can be achieved by increasing the gains of the stabilizing controller, the system response may still suffer by the low stability margin, i.e., the poles which are close to imaginary axis in Fig. 5b. Furthermore, the energy consumption highly increases as the gains of the stabilizing controller are increased (see Fig. 6).

Let us now consider the DOB-based robust control of the unstable plant. Fig. 7 illustrates the pole spread of the robust control system when DOB is used to suppress disturbances due to parametric uncertainties in the inner-loop and Eq. (41) is used to stabilize the outer-loop. It is clear from the figure that the robust stability can be achieved by using Eq. (41) when DOB 


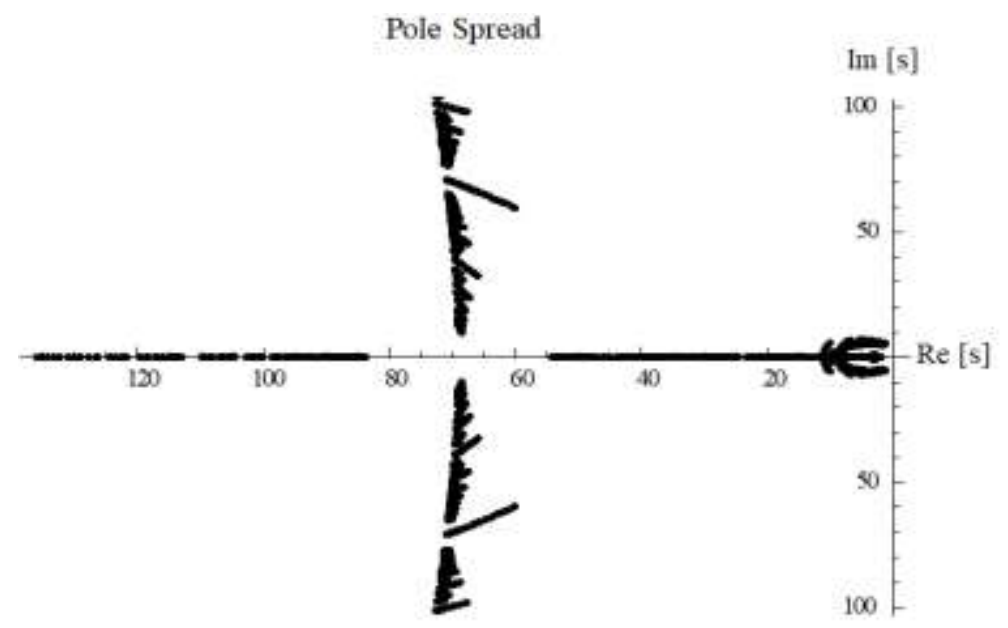

Fig. 7. Pole spread of the robust control system. The outer-loop stabilizing controller is $C_{F B}(s)=10+6 s$ and the bandwidth of DOB is $100 \mathrm{rad} / \mathrm{s}$.

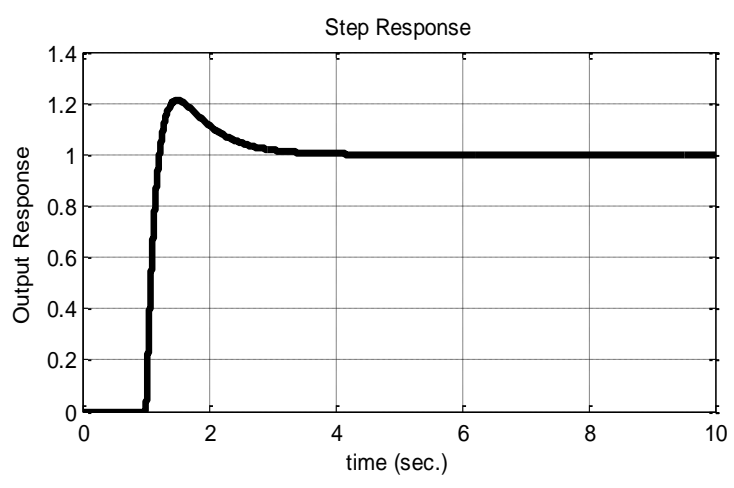

a) $C_{F F}(s)=1$.

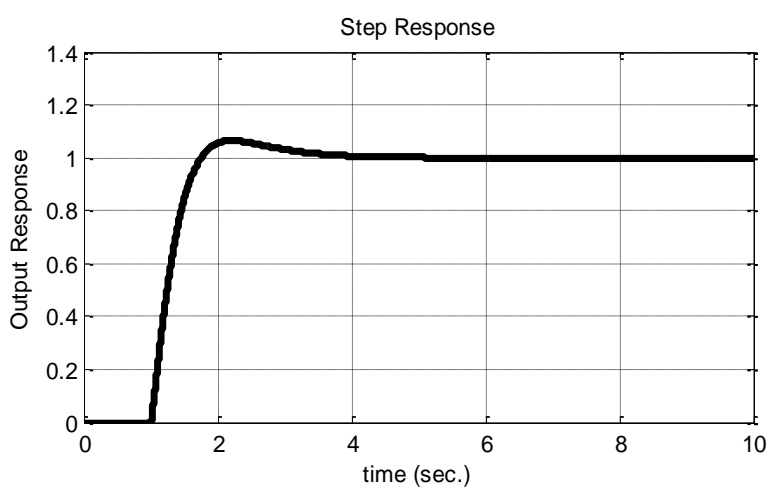

b) $C_{F F}(s)=\frac{s+10}{4 s+10}$

Fig. 8. Step responses of the robust control system when the plant has an uncertain RHP pole.

is implemented in the inner-loop. As the bandwidth of DOB is increased, the poles get closer to their nominal values.

However, the bandwidth of DOB cannot be freely increased in real applications.

Fig. 8 illustrates the step responses of the robust control system when it is disturbed by not only parametric uncertainties but also $\tau_{d}=\sin (5 t)+u(t-2)$. The proposed robust controller can precisely follow step reference input by cancelling disturbances via DOB. The overshoot of the system response can be suppressed by using the proposed feed-forward controller. Fig. 6 and Fig. 8 show that DOB-based robust control system can suppress disturbances by using low control signals. Therefore, it is more energy efficient than high gain robust control methods.

Fig. 9 illustrates the frequency responses of the sensitivity and co-sensitivity functions of the outer-loop when the bandwidth of DOB has different values. As it is increased, the peak values of the sensitivity and co-sensitivity functions are lessened; i.e., the robust stability and performance of the system are improved. 
Bode Diagram

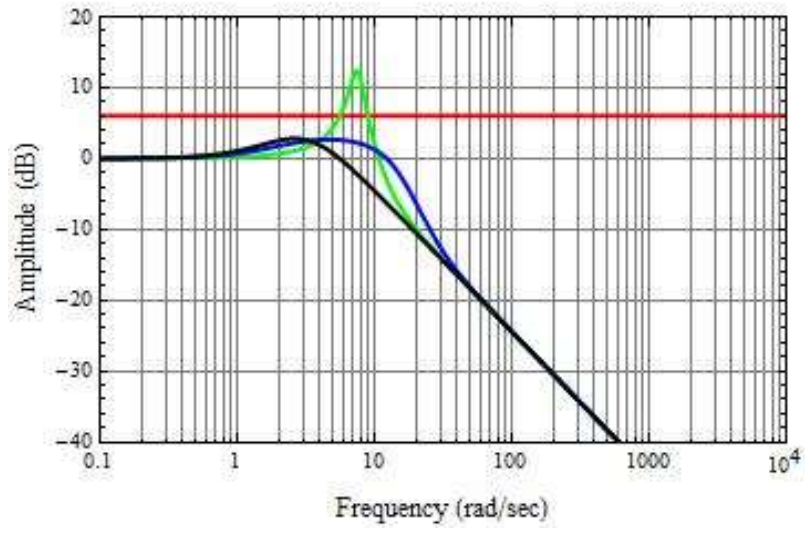

a) Co-Sensitivity function responses
Bode Diagram

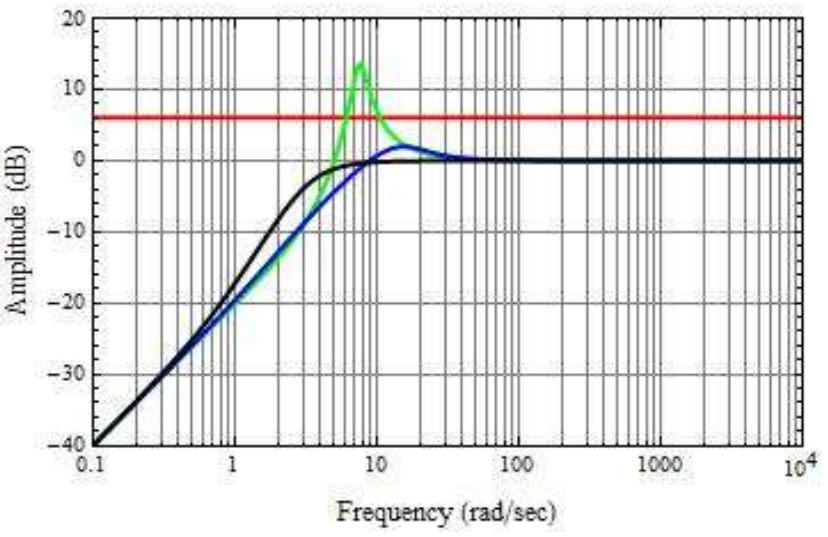

b) Sensitivity function responses

Fig. 9. Frequency responses of the outer-loop sensitivity and co-sensitivity functions. Red curves: Bounds of sensitivity and co-sensitivity functions, i.e., $|S(j w)| \leq 2$ and $|T(j w)| \leq 2$; green curves: $\mathrm{g}=10 \mathrm{rad} / \mathrm{s}$; blue curves: $\mathrm{g}=30 \mathrm{rad} / \mathrm{s}$; black curves: $\mathrm{g}=100 \mathrm{rad} / \mathrm{s}$.

Fig. 10 illustrates the stability margin of the robust control system when the bandwidth of DOB has different values. It is plotted by using Tsypkin-Polyak theorem [26, 27]. In this figure, unit circle represents the boundary of stability and the minimum distance between the unit circle and a curve represents the robust stability margin. Fig. 10 shows that the stability margin of the roust control system improves as the bandwidth of DOB is increased.

\section{CONCLUSION}

This paper has proposed DOB-based robust controllers for plants with RHP zero(s) and pole(s). In DOB-based control, the robustness against disturbances is directly related to the bandwidth of the LPF of DOB. However, its bandwidth cannot be freely tuned if plants have RHP pole(s) and zero(s). It is shown in this paper that if the plant is non-minimum phase, then the

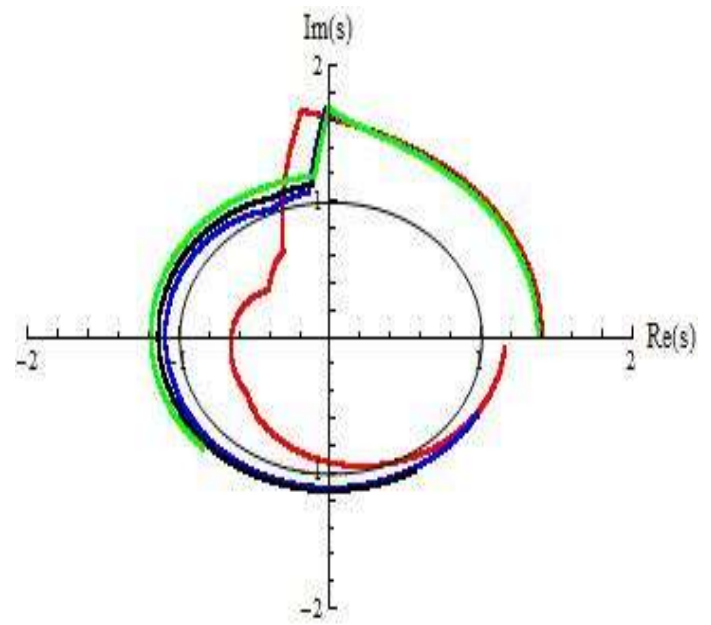

Fig. 10. Stability margin of the DOB based robust control system. Red curve: $\mathrm{g}=10 \mathrm{rad} / \mathrm{s}$; blue curve: $\mathrm{g}=52 \mathrm{rad} / \mathrm{s}$; black curve: $\mathrm{g}=100 \mathrm{rad} / \mathrm{s}$; green curve: $\mathrm{g}$ $=250 \mathrm{rad} / \mathrm{s}$. 
bandwidth of DOB has an upper bound to achieve robust stability; however, if the plant is unstable, then the robust stability can be achieved when the bandwidth of DOB is set at higher than its lower bound. The bandwidth limitation becomes more severe if the plant has RHP zero(s) and pole(s) and they are close each other. The upper and lower bounds are analytically derived by using Poisson integral formula in Theorem 2 and Theorem 3. Although the analytical bounds suffer from conservatism, they provide basic insights regarding the bandwidth limitations of DOB when plants have RHP zero(s) and pole(s). The internal stability problem is tackled by proposing a minimum phase approximation of the non-minimum phase nominal plant model within a limited frequency range. This frequency range is determined by the RHP zero of the plant. The validity of proposals is verified by giving simulation results.

\section{REFERENCES}

[1] L. Qiu, E. J. Davison, "Performance limitations of non-minimum Phase Systems in the Servomechanism Problem,” Automatica, vol. 29, no.2, pp. 337349,1993

[2] J.S. Freudenberg, D.P. Looze, "Right half plane poles and zeros and design tradeoffs in feedback systems," IEEE Trans. on Automatic Control, vol. 30, no. 6, pp. 555-565, June 1985.

[3] http://www.ieeecss-oll.org/lectures/1989/respect-unstable

[4] G. Stein, "Respect the Unstable," IEEE Control Systems Magazine, vol. 23, no. 4, pp. 12- 25, Aug.2003.

[5] G. C. Goodwin, M. E. Salgado, J. I. Yuz, "Performance Limitations for Linear Feedback Systems in the Presence of Plant Uncertainty," IEEE Trans. on Automatic Control, vol. 48, no. 8, Aug. 2003.

[6] T. Umeno, Y. Hori, "Robust speed control of dc servomotors using modern two degrees-of-freedom controller design," IEEE Transactions on Industrial Electronics, vol. 38, no. 5, Oct. 1991.

[7] K. Ohnishi, M. Shibata, T. Murakami, "Motion Control for Advanced Mechatronics," IEEE/ASME Trans. Mechatronics, vol. 1, no.1, March 1996.

[8] E. Schrijver, D. V. Johannes, "Disturbance Observers for Rigid Mechanical Systems: Equivalence, Stability, and Design,” Journal of Dynamic Systems, Measurement, and Control, vol.124, no 4, pp. 539-548, Dec. 2002

[9] L. Guo, M. Tomizuka, "High-speed and high-precision motion control with an optimal hybrid feed forward controller," IEEE/ASME Trans Mechatronics, vol. 2, no. 2, pp.110-122, June 1997.

[10] G. Zhao, Y. Zhao, L. Zhang, "Research on Observer and Compensator for Noise Disturbances in CNC Servo System," International Conference on Intelligent Computation Technology and Automation (ICICTA), vol.3, pp. 53-56, 2010.

[11] B. A. Guvenc, L. Guvenc, S. Karaman, "Robust MIMO disturbance observer analysis and design with application to active car steering," Int. J. Robust Nonlinear Control, vol. 20, pp. 873-891, 2010

[12] S. P. Chan, "A disturbance observer for robot manipulators with application to electronic components assembly," IEEE Transactions on Industrial Electronics, vol. 42, no. 5, pp. 487-493, Oct. 1991.

[13] J. Yang, W. H. Chen, S. Li, L. Guo and Y. Yan, "Disturbance/Uncertainty Estimation and Attenuation Techniques in PMSM Drives-A Survey," in IEEE Transactions on Industrial Electronics, vol. 64, no. 4, pp. 3273-3285, April 2017.

[14] E. Sariyildiz, K. Ohnishi, "Robust stability and performance analysis of the control systems with higher order disturbance observer: Frequency approach," International Conference on Human System Interaction (HSI), Perth Australia, 6-8 June 2012.

[15] E. Sariyildiz, K. Ohnishi, "Design constraints of disturbance observer in the presence of time delay," IEEE International Conference on Mechatronics (ICM), Vicenza, Italy, Feb. 2013.

[16] E. Sariyildiz, K. Ohnishi, "A new solution for the robust control problem of non-minimum phase systems using disturbance observer," IEEE International Conference on Mechatronics (ICM), Vicenza, Italy, February 2013.

[17] W. C. Yang, M. Tomizuka, "Disturbance rejection through an external model for non-minimum phase systems", ASME J. of Dynamic Systems, Measurement and Control, Vol. 116 No. 1, pp. 39-44, 1994.

[18] N. H. Jo, H. Shim, Y. I. Son, "Disturbance observer for non-minimum phase linear systems", International Journal of Control, Automation, and Systems (2010) 8(5):994-1002

[19] X. Chen, G. Zhai, and T. Fukuda, "An approximate inverse system for non-minimum-phase systems and its application to disturbance observer," Systems \& Control Letters, vol. 52, pp. 193-207, 2004.

[20] M. M. Seron, J. H. Braslavsky and G. C. Goodwin, "Fundamental Limitations in Filtering and Control," Springer, ISBN 978-1-4471-0965-5.

[21] H.W. Bode, "Network Analysis and Feedback Amplifier Design," D. van Nostrand, NewYork, 1945

[22] R.H. Middleton, "Trade-offs in linear control system design," Automatica, vol. 27, pp. 281-292, 1991.

[23] N. Wiener, P. Masani, "The prediction theory of multivariate stationary stochastic processes," Acta Math., vol. 98, pp. 11-150, 1957.

[24] D. E. Goldberg, "Genetic algorithms in search, optimization and machine learning," Addison-Wesley Longman Publishing Co., Inc. Boston, MA, USA, 1989 .

[25] C. R. Reeves, J. E. Rowe, “Genetic algorithms - principles and perspectives: A guide to genetic algorithm theory,” Springer, 2002.

[26] Y. Z. Tsypkin, B. T. Polyak, "Frequency domain criterion for robust stability of polytope of polynomials," In: Mansour, M., Balemi, S., Truol, W. (eds.) Control of Uncertain Dynamic Systems, pp. 113-124. Birkhauser, Basel, 1992.

[27] E. Sariyildiz, K. Ohnishi, "Bandwidth constraints of disturbance observer in the presence of real parametric uncertainties," Eur. J. Control, May 2013, vol. 19, Issue 3, pp.199-205. 